\title{
Investigating the electro-optical properties of non-stoichiometric silicon nitride thin films for photovoltaic applications
}

\section{Investigando las propiedades electro-ópticas de capas delgadas de nitruro de silicio no estequiométrico para aplicaciones fotovoltaicas}

\author{
Oriol Blázquez(1), Julià López-Vidrier(1,*), Sergi Hernández(1), \\ Josep Montserrat( ${ }^{(2)}$, Blas Garrido(1) \\ 1. Department d'Electrònica, Universitat de Barcelona, C/ Martí i Franquès 1, 08028 Barcelona, Spain. \\ 2. IMB-CNM, CSIC, Bellaterra, 08193 Barcelona, Spain. \\ ${ }^{(*)}$ Email: jlopezv@el.ub.edu \\ Received / Recibido: 07/06/2013. Revised / Revisado: 19/09/2013. Accepted / Aceptado: 07/10/2013. \\ DOI: $\underline{\text { http://dx.doi.org/10.7149/OPA.46.4.309 }}$
}

\begin{abstract}
:
The electro-optical properties of devices containing Si-rich silicon nitride (SRN) thin films, deposited by means of low-pressure chemical-vapor deposition (LPCVD), are studied. After optically evidencing the presence of defects in our films, a Poole-Frenkel conduction mechanism has been considered, which is in agreement with electrical experiments. We found that the poly-Si layer strongly modulates the electroluminescence and light absorption of the devices. In addition, they showed photocurrent and photoconductivity under illumination, SRN becoming a promising material for photovoltaic applications.
\end{abstract}

Key words: Si-Rich Nitride, LPCVD, PL, Poole-Frenkel Transport Mechanism, EL, Photoconductivity, Photocurrent.

\section{RESUMEN:}

Se han estudiado las propiedades electro-ópticas de dispositivos con capas delgadas de nitruro de silicio rico en silicio (SRN, de sus siglas en inglés), fabricadas mediante un depósito químico de vapor a baja presión (LPCVD). Después de la evidencia óptica de la presencia de defectos en dichas capas, se consideró un mecanismo de conducción del tipo Poole-Frenkel, de acuerdo con los experimentos eléctricos. Se observó que la capa de poli-Si modula fuertemente la electroluminiscencia y la absorción de luz de los dispositivos. Además, éstos mostraron fotocorriente y fotoconductividad bajo iluminación, lo que hace del SRN un material prometedor para aplicaciones fotovoltaicas.

Palabras clave: Nitruro Rico en Silicio, LPCVD, PL, Mecanismo de Transporte Poole-Frenkel, EL, Fotoconductividad, Fotocorriente.

\section{REFERENCES AND LINKS / REFERENCIAS Y ENLACES}

[1]. L. T. Canham, "Silicon quantum wire array fabrication by electrochemical and chemical dissolution of wafers", Appl. Phys. Lett. 57, 1046-1048 (1990). DOI

[2]. G. Conibeer, M. Green, E.-C. Cho, D. König, Y.-H. Cho, T. Fanguwannarak, G. Scardera, E. Pink, Y. Huang, T. Puzzer, S. Huang, D. Song, C. Flynn, S. Park, X. Hao, D. Mandfield, "Silicon quantum dot nanostructures for tandem photovoltaic cells", Thin Solid Films 516, 6748-6756 (2008). DOI

[3]. M. Zacharias, J. Heitmann, R. Scholz, U. Kahler, M. Schmidt, J. Bläsing, "Size-controlled highly luminescent silicon nanocrystals: A SiO/SiO² superlattice approach", Appl. Phys. Lett. 80, 661-663, (2002). DOI

[4]. J. López-Vidrier, S. Hernández, A. M. Hartel, D. Hiller, S. Gutsch, P. Löper, L. López-Conesa, S. Estradé, F. Peiró, M. Zacharias, B. Garrido, "Structural and optical characterization of size controlled silicon nanocrystals in $\mathrm{SiO}_{2} / \mathrm{SiO}_{x} \mathrm{~N}_{y}$ multilayers", Energy Procedia 10, 43-46 (2011). DOI 
[5]. J. Barreto, M. Perálvarez, A. Morales, B. Garrido, J. Montserrat, C. Domínguez, "Broad range adjustable emission of stacked $\mathrm{SiN}_{x} / \mathrm{SiO}_{y}$ layers", J. Mater. Res. 23, 1513-1516 (2008). DOI

[6]. J. Tauc, "Optical properties and electronic structure of amorphous Ge and Si”, Mater. Res. Bulletin 3, 37-46 (1968). DOI

[7]. R. Guerra, M. Ippolito, S. Meloni, S. Ossicini, "The influence of silicon nanoclusters on the optical properties of a-SiN ${ }_{x}$ samples: A theoretical study", Appl. Phys. Lett. 100, 181905-181908 (2012). DOI

[8]. N. Murgatroyd, "Theory of space-charge-limited current enhanced by Frenkel effect", J. Phys. D: Appl. Phys. 3, 151-156 (1970). DOI

[9]. Y. Berencén, J. M. Ramírez, O. Jambois, C. Domínguez, J. A. Rodríguez, B. Garrido, “Correlation between charge transport and electroluminescence properties of Si-rich oxide/nitride/oxide-based light emitting capacitor", J. Appl. Phys. 112, 033114-033118 (2012). DOI

\section{Introducción}

Actualmente, las células solares son uno de los dispositivos electro-ópticos más interesantes, gracias a la posibilidad de obtener energía mediante fuentes no tóxicas e inagotables. Durante las dos últimas décadas, se han estudiado las propiedades relacionadas con el tamaño de los nanocristales de silicio [1]. Debido a que los nanocristales muestran una mayor eficiencia en la absorción de fotones de alta energía del espectro solar [2], es posible aumentar la eficiencia de los dispositivos fotovoltaicos basados en silicio.

Muchos estudios se han centrado en nanocristales de silicio embebidos en matrices de óxido de silicio $[3,4]$. Sin embargo, la elevada energía de banda prohibida del $\mathrm{SiO}_{2}(9 \mathrm{eV})$, supone un serio problema para el transporte eléctrico [2]. Por otra parte, el nitruro de silicio presenta una menor energía de banda prohibida (5.3 eV), la cual permite una mejor conducción de portadores $\mathrm{y}$, por consiguiente, un mejor rendimiento opto-electrónico [2]. En este trabajo, estudiamos el comportamiento óptico, eléctrico y electro-óptico de capas delgadas de nitruro de silicio rico en silicio (SRN), en función del exceso de silicio.

\section{Detalles experimentales}

Capas de $50 \mathrm{~nm}$ de $\mathrm{Si}_{3} \mathrm{~N}_{4}$ fueron depositadas sobre sustratos de $\mathrm{Si}$ cristalino (c-Si) y vidrio mediante un depósito químico de vapor a baja presión (LPCVD, de sus siglas en inglés), utilizando $\mathrm{SiH}_{4}$ y $\mathrm{NH}_{3}$ como gases precursores (ver Ref. [5] para más detalles). Después del depósito, se llevó a cabo una implantación iónica de Si con una energía de $28 \mathrm{keV}$, bajo un ángulo de $7^{\circ}$ y utilizando dosis de implantación de $1.9 \times 10^{16}$ y $2.5 \times 10^{16} \mathrm{at} \cdot \mathrm{cm}^{-2}$ para lograr unos excesos de Si del 16 y 20 at.\%, respectivamente, quedando una distribución Gaussiana dentro de la capa de nitruro. Posteriormente, las muestras fueron sometidas a un recocido a presión ambiente en una atmósfera inerte de $\mathrm{N}_{2}$, utilizando temperaturas entre 950 y $1100^{\circ} \mathrm{C}$ durante 1 hora. A partir de algunas muestras depositadas sobre c-Si, y con un recocido de $950^{\circ} \mathrm{C}$, se fabricaron dispositivos metalizando con Al la parte inferior y con $100 \mathrm{~nm}$ de poli-Si la parte superior, siendo esta última capa fuertemente dopada tipo $n\left(10^{18} \mathrm{~cm}^{-3}\right)$ con $\mathrm{POCl}_{3}$. La estructura final de los dispositivos se muestra en la Fig. 1.

Los espectros de fotoluminiscencia (PL) fueron adquiridos utilizando un monocromador acoplado a un tubo foto-multiplicador de GaAs y utilizando técnicas estándar de filtrado. La excitación fue llevada a cabo mediante fotones de longitud de onda de $325 \mathrm{~nm}$ de láser de HeCd. Se realizaron medidas de reflectancia $y$ transmitancia (R\&T) utilizando la esfera

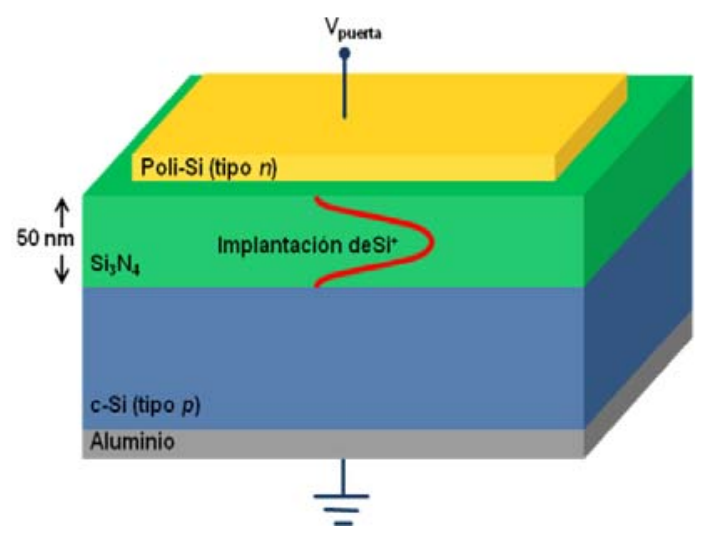

Fig. 1. Esquema de la sección transversal de los dispositivos empleados. 
integradora de un sistema de respuesta fotovoltaica Bentham PVE300, detectando la señal con un detector de InGaAs en un rango entre 300 y $1100 \mathrm{~nm}$.

La caracterización eléctrica en oscuridad se realizó mediante un sistema de puntas Microtech 11000 equipado con un analizador de dispositivos semiconductores Agilent B1500. El sistema permitió hacer medidas en tempe-ratura en un rango entre 25 y $300^{\circ} \mathrm{C}$. Por una parte, una CCD enfriada con nitrógeno líquido y acoplada a un monocromador permitió la obtención de los espectros de electroluminiscencia (EL) de los dispositivos, excitando éstos últimos con una corriente constante de $500 \mu \mathrm{A}$ en régimen de acumulación. Por otra parte, se midió la fotorespuesta iluminando los dispositivos con una combinación de lámparas de Xe y halógena de cuarzo y un monocromador, contenidos en el sistema PVE300, permitiendo fotoexcitar los dispositivos bajo estudio mediante longitudes de onda discretas. También se utilizó una lámpara de cuarzo halógena para simular el espectro solar AM1.5G.

\section{Resultados y discusión}

\subsection{Propiedades ópticas}

Las medidas de PL se llevaron a cabo utili-zando las muestras sobre substrato de c-Si y con diferentes temperaturas de recocido. La Fig. 2(a) presenta el espectro de PL de la muestra con un 20 at.\% de exceso de Si y recocida a $950^{\circ} \mathrm{C}$. Se observa una clara emisión en el rango visible con un máximo entorno a $2 \mathrm{eV}$. Además, se apreció una fuerte dependencia con la temperatura de recocido, presentando la muestra con mayor temperatura de recocido un aumento de casi el $50 \%$ en intensidad que la recocida a menor temperatura.

Medidas de R\&T se realizaron sobre muestras sobre vidrio. El espectro resultante se corrigió por la transmitancia del sustrato para obtener únicamente la contribución de la capa de SRN. El coeficiente de absorción $(\alpha)$ fue calculado usando:

$$
\alpha(\lambda)=\frac{1}{d} \ln \left(\frac{T_{s}(\lambda)[1-R(\lambda)]}{T(\lambda)}\right),
$$

donde $d$ es el espesor de la capa activa y $T_{S}$ la transmitancia del sustrato. La energía de la banda prohibida óptica $\left(E_{g}^{o p t}\right)$ fue estimada a partir de la ley de Tauc [6]:

$$
\alpha(h v)=\frac{B}{h v}\left(h v-E_{g}^{o p t}\right),
$$

siendo $B$ una constante. La representación de $(\alpha h v)^{1 / 2}$ en función de $h v$, se presenta en la Fig. 2(b) para las muestras con 16 y 20 at.\% de exceso de silicio, depositadas sobre vidrio $\mathrm{y}$ recocidas a $950^{\circ} \mathrm{C}$. Ajustando la región lineal de altas energías mediante la Ec. (2), es posible estimar la energía del frente de absorción del sistema (i.e., la energía de la banda prohibida óptica). Se obtuvo un valor aproximado de $2.4 \mathrm{eV}$ para ambos excesos de $\mathrm{Si}$, siendo dicha energía mayor que la del pico de PL $\left(E_{g}^{e m i}<E_{g}^{o p t}\right)$, lo que se traduce en un desplazamiento de Stokes de aproximadamente $400 \mathrm{meV}$.

Debido a la alta energía de banda prohibida del $\mathrm{Si}_{3} \mathrm{~N}_{4}(5.3 \mathrm{eV})$ y el hecho de que no se
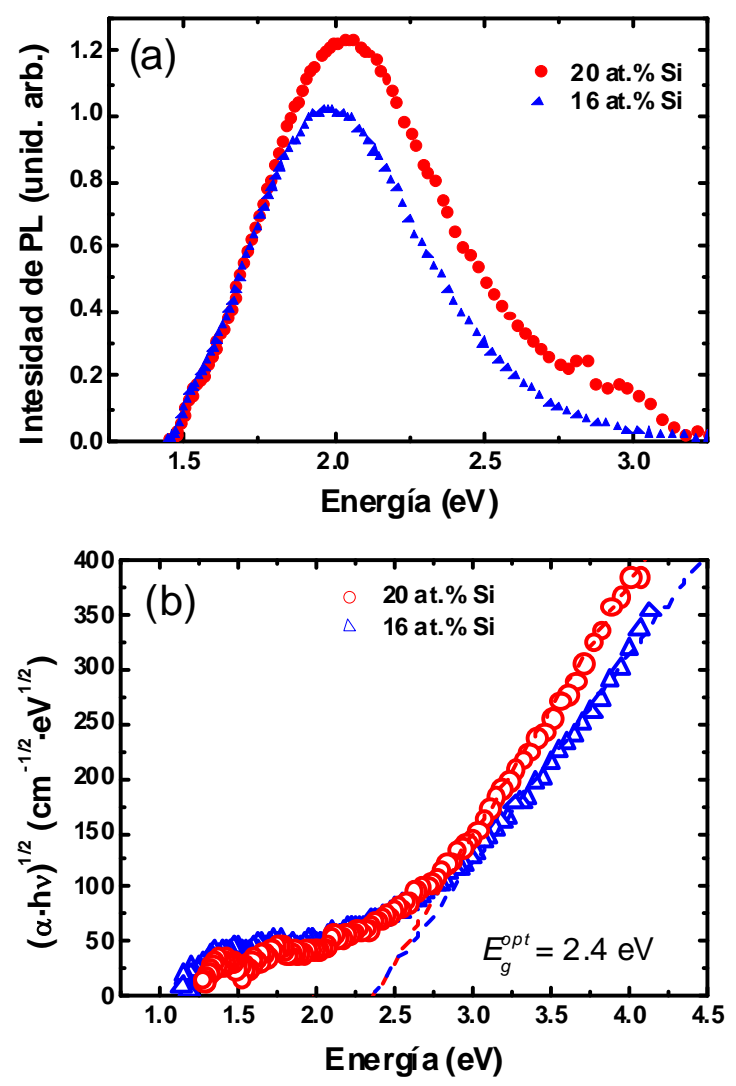

Fig. 2. (a) Espectro de PL y (b) representación de Tauc para las muestras recocidas a $950^{\circ} \mathrm{C}$ con 16 y 20 at. $\%$ de exceso de $\mathrm{Si}$. 
observa una variación destacable de las propiedades ópticas a diferentes excesos de Si y bajo diferentes temperaturas de recocido (las variaciones observadas fueron inferiores a 50 $\mathrm{meV}$ ), se planteó la hipótesis que la emisión intra-banda observada proviene de los defectos contenidos en la capa de SRN. Esta suposición está de acuerdo con Guerra et al., quienes atribuyeron esta emisión a la recombinación radiativa de defectos presentes en la interfaz $\mathrm{Si} / \mathrm{Si}_{3} \mathrm{~N}_{4}$ [7]. Además, el aumento de la intensidad de la señal de PL a mayores temperaturas de recocido se puede interpretar como una activación térmica de dichos defectos.

\subsection{Transporte eléctrico}

La caracterización eléctrica se llevó a cabo en dispositivos cuyas capas de SRN fueron recocidas a $950^{\circ} \mathrm{C}$. En la Fig. 3 se presentan las curvas $J(V)$ obtenidas en oscuridad para los diferentes excesos de $\mathrm{Si}$, apreciándose un claro aumento de la conducción al aumentar éste último. Además, se observa un comportamiento asimétrico (no mostrado en el gráfico) entre los regímenes de acumulación $(V<0)$ e inversión $(V>0)$ que sugiere una conducción limitada por los portadores minoritarios. Por esta razón, los resultados presentados de ahora en adelante corresponden al régimen de acumulación, donde los electrones son los portadores mayoritarios.

Debido a que los resultados ópticos evidencian un papel importante de los defectos en la matriz, se consideró un mecanismo de conducción del tipo Poole-Frenkel (PF), a través

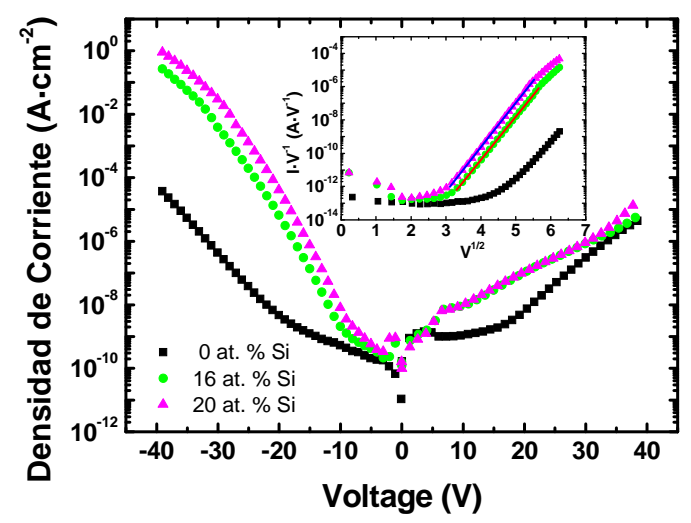

Fig. 3. Curvas $J(V)$ para diferentes excesos de Si. El recuadro superior muestra las correspondientes representaciones $\ln \left(I \cdot V^{-1}\right)$ en función de $V^{1 / 2}$ (Poole-Frenkel), ajustadas mediante la Ec. (3). de trampas que generan niveles energéticos permitidos dentro de la banda prohibida del $\mathrm{Si}_{3} \mathrm{~N}_{4}$. Según la teoría de $\mathrm{PF}$, la intensidad de corriente $I$ se puede expresar como [8]:

$$
I \propto V \exp \left(-\frac{q}{k_{B} T}\left[\phi_{0}-\sqrt{\frac{q V}{\pi \varepsilon_{0} \varepsilon_{r} d}}\right]\right),
$$

siendo $V$ el voltaje aplicado, $q$ la carga elemental, $k_{B}$ la constante de Boltzmann, $T$ la temperatura, $\varepsilon_{0}$ la permitividad en el vacío, $\varepsilon_{r}$ la permitividad relativa del medio y $d$ el espesor de la capa activa. En relación a las medidas en función de $V$ (ver Fig. 3), realizadas a temperatura ambiente, se ha representado $\ln \left(I \cdot V^{-1}\right)$ en función de $V^{1 / 2}$ (ver gráfica insertada en la Fig. 3), lo que se conoce como la representación de Poole-Frenkel. Los resultados se presentan en el recuadro superior de la Fig. 3, mostrando una región lineal a lo largo de más de cuatro órdenes de magnitud. Del valor de la pendiente obtenida mediante los ajustes lineales se ha podido estimar $\varepsilon_{r}$ para los diferentes excesos de silicio. Los valores encontrados escalan perfectamente con el exceso de silicio, encontrando $\varepsilon_{r}=7.3$ y 7.5 para excesos de silicio del $16 \%$ y $20 \%$, respectivamente [9].

Por otra parte, fue evaluada la de-pendencia con la temperatura de la corriente (ver Ec. (3)), para diferentes voltajes. En este caso la expresión corresponde a la bien conocida ley de Arrhenius:

$$
\sigma(T) \propto \exp \left(-\frac{E_{A}}{k_{B} T}\right),
$$

donde $E_{A}$ se define como la energía de activación de dicho mecanismo de conducción.

En la Fig. 4 se muestran las representaciones de Arrhenius para el dispositivo con un exceso de $\mathrm{Si}$ del 20 at.\%, obtenidas para diferentes voltajes aplicados. La evolución observada de la conductividad con la temperatura proporciona un argumento sólido para la elección del mecanismo de conducción propuesto. Mediante el ajuste de los datos experimentales a la Ec. (4), se determinaron unos valores de $E_{A}$ desde 150 a $350 \mathrm{meV}$ para voltajes decrecientes.

De acuerdo con estos resultados, se puede considerar el sistema como un conjunto de 


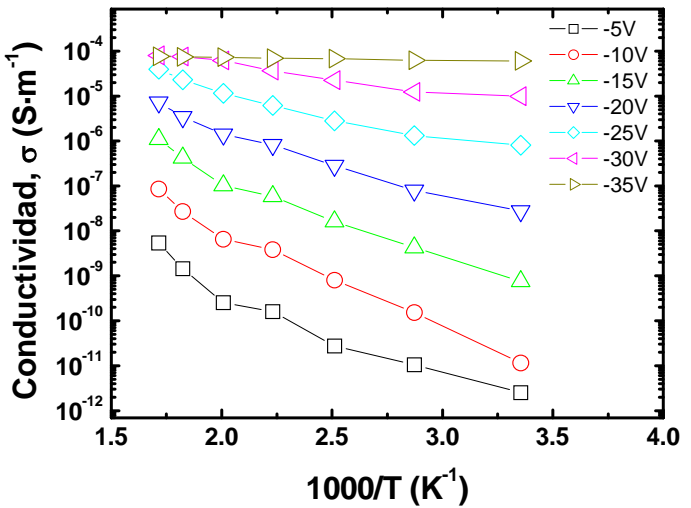

Fig. 4. Representaciones de Arrhenius para distintos voltajes aplicados sobre el dispositivo con un exceso de Si del 20 at. $\%$.

trampas con estados superficiales dentro de la banda prohibida del $\mathrm{Si}_{3} \mathrm{~N}_{4}$. Al aumentar $V$, la estructura de bandas se modifica disminuyendo la barrera de potencial para que los portadores escapen de las trampas. Por otra parte, un aumento de la $T$ eleva la energía de los portadores dentro de las trampas. Ambos efectos inducen la reducción de la barrera efectiva que los portadores (principalmente electrones) deben superar para participar en la conducción. En resumen, tanto la dependencia con la temperatura como con el voltaje sugieren un transporte de portadores activado térmicamente y asistido por trampas a través del material, en concordancia con la teoría PF.

\subsection{Propiedades electro-ópticas}

La señal de EL emitida por los dispositivos fue estudiada en el rango visible e in-frarrojo cercano, tal como se muestra en la Fig. 5, donde se compara con la emisión de PL anteriormente presentada. El pico de emisión de EL, centrado en $1.8 \mathrm{eV}$, está claramente desplazado hacia menores energías con respecto a la señal de PL $(2 \mathrm{eV})$. Para aclarar el origen de este desplazamiento, se estudió la transmitancia del contacto superior, poli-Si (recuadro de la Fig. 5), la cual presenta una dependencia con la longitud de onda, lo que dota al espectro de emisión de una modulación debida al contacto. Una vez sustraída la contribución del poli-Si al espectro de EL, ambos espectros, EL y PL, coinciden en muy buena aproximación. Además, el hecho de que con EL se recuperen los mismos

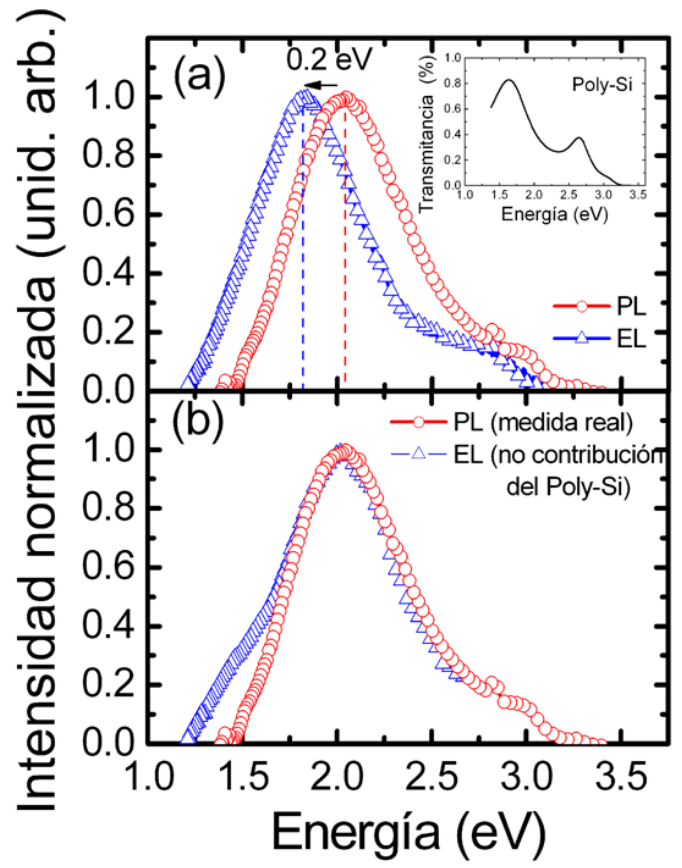

Fig. 5. Espectros de PL (círculos rojos) y EL (triángulos azules) correspondientes a las muestras con un 20 at.\% de exceso de $\mathrm{Si}$ re-cocidas a $950^{\circ} \mathrm{C}$. El recuadro presenta el espectro de transmitancia de la capa de poli-Si.

resultados que en la caracterización óptica, indica que el origen de la emisión proviene también de los defectos, lo cual está de acuerdo con el mecanismo de transporte asistido por trampas (defectos) previamente determinado y reportado en otros estudios previos [9].

La respuesta eléctrica de los mismos dispositivos bajo excitación óptica también fue investigada. En particular, se obtuvieron curvas $J(V)$ en acumulación utilizando condiciones de oscuridad y bajo iluminación de 1 sol (espectro AM1.5G), que se presentan en la Fig. 6(a). Bajo esta iluminación, se observó un claro aumento de la densidad de corriente, presentando un alta variación de la conductividad con respecto a la medida en oscuridad (es decir, fotoconductividad). Esto se atribuye a un aumento de la concentración de portadores mayoritarios (electrones) que son fotogenerados mediante las condiciones de iluminación utilizadas.

También se aplicó una luz monocromática para estudiar la dependencia espectral de la conversión fotón-electrón. El recuadro de la Fig. 6 muestra la fotocorriente normalizada al flujo 


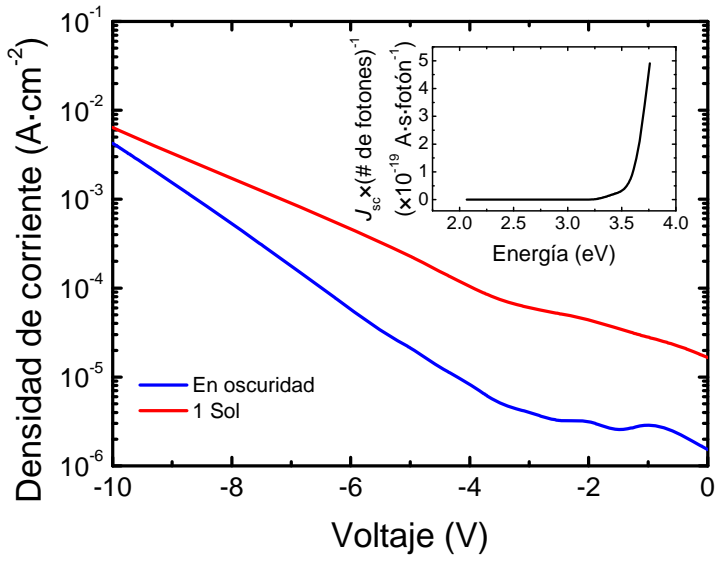

Fig. 6. Característica $J(V)$ del dispositivo con un exceso de silicio del 20 at. \%, en oscuridad y bajo condiciones de iluminación de 1 sol. El recuadro muestra la densidad de corriente en cortocircuito $\left(J_{s c}\right)$ normalizada por el flujo de fotones, en función de la energía de excitación de éstos últimos.

de fotones incidente, obtenida a $0 \mathrm{~V}$ y corregida por la transmitancia del poli-Si. El espectro resultante muestra un comportamiento similar al de absorción, presentando un umbral para la fotocorriente alrededor de $3 \mathrm{eV}$. Este valor se encuentra dentro del rango de alta absorción del espectro mostrado en la Fig. 2(b), lo que implica que parte de los fotones absorbidos participan en la fotogeneración de portadores.

\section{Conclusión}

Se han depositado capas delgadas de $\mathrm{Si}_{3} \mathrm{~N}_{4}$ sobre sustrato de silicio cristalino o vidrio mediante LPCVD. Se ha inducido un exceso de Si mediante implantación iónica de $\mathrm{Si}$ usando diferentes dosis. Posteriormente a la implantación, las capas fueron sometidas a un proceso térmico a diferentes temperaturas para intentar precipitar dicho exceso. Gracias a la caracterización óptica, se ha deducido que el origen de la luminiscencia y la absorción del SRN se relaciona con defectos situados en la interfaz $\mathrm{Si} / \mathrm{Si}_{3} \mathrm{~N}_{4}$, sin presencia de nanoagregados de Si. Se ha fabricado con dichas capas dispositivos simples metalizando la parte superior de las muestras con un poli-Si altamente dopado, y la inferior con Al. Los dispositivos han presentado un mecanismo de transporte del tipo Poole-Frenkel, corroborado a través de estudios en voltaje y temperatura, que está en concordancia con la naturaleza defectuosa del SRN. Los espectros de EL obtenidos están en perfecto acuerdo con la PL, una vez se tiene en cuenta la modulación causada por la transmitancia del poli-Si. Finalmente, se ha demostrado fotogeneración en dichos dispositivos, dando lugar a un aumento de la conducción en el sistema que depende fuertemente de la energía de los fotones incidentes.

\section{Agradecimientos}

Agradecemos la subvención otorgada por la convocatoria GICSERV-2013, concedida por el Centro Nacional de Microelectrónica - Instituto de Microelectrónica de Barcelona (CNM-IMB, CSIC), quienes fabricaron los dispositivos empleados en el presente estudio. O.B. agradece la financiación recibida del European Community's Seventh Framework Programme (FP7/2007-2013), en virtud del acuerdo de subvención número 245977 - proyecto NASCEnT. 